\title{
Effect of auditory constraints on motor performance depends on stage of recovery post-stroke
}

\author{
Viswanath Aluru ${ }^{1}$, Ying Lu ${ }^{2}$, Alan Leung ${ }^{3}$, Joe Verghese ${ }^{4}$ and Preeti Raghavan ${ }^{1,5}$ * \\ ${ }^{1}$ Department of Rehabilitation Medicine, New York University School of Medicine, New York, NY, USA \\ ${ }^{2}$ Center for the Promotion of Research Involving Innovative Statistical Methodology, Steinhardt School of Culture, Education and Human Development, New York \\ University, New York, NY, USA \\ ${ }^{3}$ University of Pittsburgh Medical Center, Pittsburgh, PA, USA \\ ${ }^{4}$ Department of Neurology, Albert Einstein College of Medicine, Bronx, NY, USA \\ ${ }^{5}$ Department of Physical Therapy, Steinhardt School of Culture, Education and Human Development, New York University, New York, NY, USA
}

Edited by:

Renée Morris, University of New South Wales, Australia

\section{Reviewed by:}

William Hutchison, University Health Network, Canada

Andrew Paul Tosolini, University of New South Wales, Australia

\section{${ }^{*}$ Correspondence:}

Preeti Raghavan, Department of Rehabilitation Medicine, Rusk Institute of Rehabilitation Medicine, NYU Langone Ambulatory Care Center, 240 E 38th Street, 17th Floor New York, NY 10016, USA

e-mail: preeti.raghavan@nyumc.org
In order to develop evidence-based rehabilitation protocols post-stroke, one must first reconcile the vast heterogeneity in the post-stroke population and develop protocols to facilitate motor learning in the various subgroups. The main purpose of this study is to show that auditory constraints interact with the stage of recovery post-stroke to influence motor learning. We characterized the stages of upper limb recovery using task-based kinematic measures in 20 subjects with chronic hemiparesis. We used a bimanual wrist extension task, performed with a custom-made wrist trainer, to facilitate learning of wrist extension in the paretic hand under four auditory conditions: (1) without auditory cueing; (2) to non-musical happy sounds; (3) to self-selected music; and (4) to a metronome beat set at a comfortable tempo. Two bimanual trials ( $15 \mathrm{~s}$ each) were followed by one unimanual trial with the paretic hand over six cycles under each condition. Clinical metrics, wrist and arm kinematics, and electromyographic activity were recorded. Hierarchical cluster analysis with the Mahalanobis metric based on baseline speed and extent of wrist movement stratified subjects into three distinct groups, which reflected their stage of recovery: spastic paresis, spastic co-contraction, and minimal paresis. In spastic paresis, the metronome beat increased wrist extension, but also increased muscle co-activation across the wrist. In contrast, in spastic co-contraction, no auditory stimulation increased wrist extension and reduced co-activation. In minimal paresis, wrist extension did not improve under any condition. The results suggest that auditory task constraints interact with stage of recovery during motor learning after stroke, perhaps due to recruitment of distinct neural substrates over the course of recovery. The findings advance our understanding of the mechanisms of progression of motor recovery and lay the foundation for personalized treatment algorithms post-stroke.

Keywords: bimanual movements, upper extremity, rehabilitation, motor learning/training, electromyography, task specificity, cerebrovascular disorders

\section{INTRODUCTION}

Stroke strikes one in six people worldwide. It is the leading cause of disability in the United States (1) and Europe (2), and the second leading cause of disability in the world (3). Hemiparesis is the most common reason for stroke-related disability, and the majority of individuals with hemiparesis have persistent deficits in hand function (4). There has been a recent surge in the availability of new rehabilitation strategies post-stroke. However, several large randomized controlled trials have failed to show the benefit of any one intervention over conventional treatment (5), and there remains a lack of understanding about how to select an appropriate treatment strategy for a given individual. While it is now accepted that task-specific training is an important aspect of a rehabilitation intervention, the constraints under which the task(s) should be practiced to be optimally therapeutic are not known. A constraint may be defined as the specific conditions under which a task is performed, for example, with one hand or both, with auditory/visual/multi-sensory feedback or without, etc. Task constraints are important because they regulate the information that is processed and assimilated by the nervous system, and the selection of constraints for any specific task may depend on the integrity and/or capacity to recruit specific neural substrates that facilitate processing of the relevant movement-related information. The stage of motor recovery, as measured by the level of motor impairment, may provide an indication for the type of task-specific constraints that are useful during practice for a given individual.

Fortunately, recovery of motor function after a hemiplegic stroke has been shown to follow a predictable pattern. Twitchell (6), Brunnstrom (7), and Fugl-Meyer et al. (8) described a hierarchical progression of recovery of patients who initially present with flaccid paralysis on one side of the body with areflexia. The 
reflex activity returns next and becomes heightened as spasticity emerges, and voluntary movements occur in stereotypical flexor and extensor synergy patterns. Spasticity then reaches its maximum level, producing characteristic patterns of stretch-sensitive responses such as spastic co-contraction. Eventually, the synergy patterns start to break up and spasticity begins to reduce as normal patterns of voluntary movement are restored. The emergence and disappearance of spasticity are thus important milestones in motor recovery $(9,10)$, although the severity of spasticity may vary considerably and temporary arrests in recovery or "plateaus" can occur at any stage (6).

Recent imaging studies further show how recovery processes unfold after a stroke [see Ref. (11) for review]. Early in recovery, the undamaged contralesional hemisphere shows increased activation (12-15), but eventually normal sensorimotor lateralization is restored in the stroke-affected hemisphere (16-18). Importantly, increases in neural activity in the contralesional motor areas in the first weeks after stroke correlate with better motor recovery in humans (19, 20) and monkeys (15), although persistent activation of the motor and non-motor areas in the contralesional hemisphere is noted in patients with poor motor outcome $(18,21)$. A recent longitudinal case study of a patient's recovery over 21 months revealed continuous change in activation in the contralesional hemisphere with concomitant improvement in motor performance, whereas the ipsilesional hemisphere demonstrated significant change only toward the end of the study period (22). Taken together, these studies suggest that (1) redundant homologous pathways in the intact hemisphere can facilitate re-organization of the central nervous system, particularly in the earlier stages of recovery, and (2) that motor recovery occurs over a protracted and variable time period post-stroke. Hence the time since stroke may not reflect where an individual is in his or her recovery process.

Two kinds of bimanual training protocols have been developed to capitalize on contralesional cortical activity post-stroke. In active bimanual training, both arms move independently and simultaneously, requiring that individuals have at least some active movement on the paretic side. Active bimanual arm training combined with rhythmic auditory stimulation (BATRAC protocol) led to increased recruitment in the contralesional and ipsilesional hemispheres with concomitant improvement in performance of the paretic hand $(23,24)$. These data suggest that there may be a synergistic effect of bimanual and auditory constraints, but their individual contribution to performance improvement has not been ascertained. Rhythmic auditory stimulation by itself has also been found to be a useful adjunct to post-stroke rehabilitation (25-28). In active-passive bimanual training, the non-paretic arm drives movements of the paretic arm and leads to simultaneous mirror movements of both arms. Here, bimanual training occurred without auditory stimulation, was used to prime the ipsilesional motor cortex for subsequent training with the paretic arm, and also led to significant gains in arm function (29-32). An advantage of the active-passive approach is that it requires little active movement in the paretic arm and can therefore be used in individuals with significant paresis. Furthermore, the active-passive approach may be used to probe subsequent motor learning with the paretic arm. We have previously shown that motor learning is often impaired with the paretic hand, but may be temporarily restored after prior practice with the non-paretic hand (33).

In this study, we sought to determine the effect of various auditory constraints on bimanual-to-unimanual (paretic hand) learning in individuals at different stages of motor recovery poststroke. Rhythmic stimulation with a metronome has been shown to improve spatiotemporal control of arm movements, perhaps via activation of brainstem-cerebellar networks $(34,35)$. However, several lines of evidence suggest that emotional drive via activation of limbic networks may also be an important predictor of motor performance (36) and post-stroke motor recovery (37). Music has been shown to activate a bilateral network of mesolimbic structures involved in processing emotions and reward information (38), and affective vocalizations have been shown to modulate attention via activation of pre-frontal-limbic networks (39). It is not clear when over the course of recovery one type of auditory stimulation versus another or no auditory stimulation will be beneficial. Hence, the objectives of this study were to: (1) characterize the stage of recovery in a disparate group of subjects with post-stroke hemiparesis using task-based kinematic measures, and (2) to examine how various types of auditory constraints interact with stage of recovery to facilitate learning with the paretic limb on a bimanual-to-unimanual learning task. Since voluntary wrist extension is frequently compromised post-stroke (40) and active wrist extension ability is predictive of hand function (41), we focused our task on training of wrist extension in the paretic hand. We hypothesized that auditory constraints that enhance emotional drive would facilitate learning of wrist extension with the paretic arm particularly in the early stages of recovery post-stroke.

\section{MATERIALS AND METHODS SUBJECTS}

Twenty subjects with chronic post-stroke hemiparesis (at least 6 months prior to enrollment) were recruited through referrals from physicians at the Rusk Institute of Rehabilitation Medicine and through public advertisement. Subjects provided informed consent in accordance with the Institutional Review Board of the New York University School of Medicine. All subjects had at least $15^{\circ}$ of passive and $5^{\circ}$ of active wrist extension on the paretic side to perform unimanual movements, and they were screened to rule out hearing deficits prior to participation.

\section{PROTOCOL}

The clinical assessments and experimental protocols were administered by well-trained research staff at the Motor Recovery Research Laboratory in the Rusk Institute of Rehabilitation Medicine. At the first visit, the Fugl-Meyer Scale (8) was used to assess upper extremity motor impairment; the Modified Ashworth Scale (42) assessed spasticity in the affected shoulder, elbow, wrist, and finger joints; active and passive range-of-motion at shoulder, elbow, wrist, and finger joints were measured using a goniometer (43), and the threshold for joint proprioception was also assessed. Depression and mood were assessed using the 15item Geriatric Depression Scale, which has been recommended for 


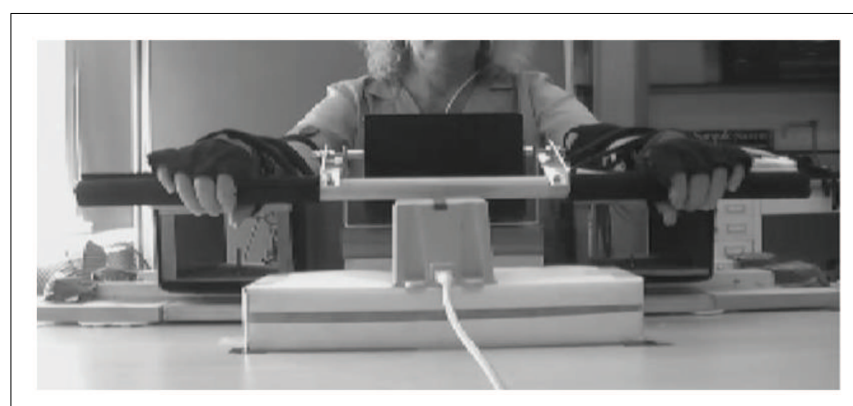

FIGURE 1 | Custom-made wrist extension trainer.

the assessment of post-stroke depression in adults of all ages (44, 45), and the Brunel Mood Scale (BRUMS) (36), respectively. An appropriate tempo for the metronome beat was then determined by asking subjects to flex and extend their paretic wrist at a comfortable pace using a custom-made wrist trainer (Figure 1) for $15 \mathrm{~s}$. Subjects then selected three familiar songs from public media to increase their feeling of vigor, happiness, and calmness. An uptempo major key song that matched their metronome speed, or was in multiples of their metronome speed, was chosen to induce a positive mood-state. The BRUMS Scale was repeated after the subjects listened to their self-selected song to verify improvement in mood-states (Figure 2).

At the second visit, subjects performed repeated bimanual and unimanual (with the paretic hand) wrist flexion-extension movements using a custom-made wrist trainer. The device was designed to constrain movement of the wrist in the sagittal plane, and limit compensatory movement of the forearm and arm. The height of the chair was adjusted to keep the shoulders level and maintain proper alignment of the trunk. Table height and the position of the wrist trainer were maintained across all task conditions for each subject. Before the start of the experiment, subjects were informed that the goal of training was to facilitate wrist extension. Electromagnetic motion sensors (trakSTAR, Ascension Technology Corporation, Shelburne, VT, USA) affixed to the limb segments on each side measured wrist kinematics. Bipolar surface electrodes (DE 2.1, Delsys Inc., Natick, MA, USA) affixed over the flexor carpi ulnaris (FCU) and extensor carpi radialis longus (ECRL) muscles on each limb recorded electromyographic (EMG) signals. Video, kinematic, and EMG data were captured synchronously using The Motion Monitor (Innovative Sports Training Inc., Chicago, IL, USA), and analysis was performed offline using Spike 2 (Cambridge Electronic Design, Cambridge, England).

Wrist movements were performed under four different auditory conditions: (1) at baseline without auditory cueing; (2) to positively valenced affective "happy" sounds (baby's laughter) recorded for $11 \mathrm{~s}$ and looped continuously, providing non-musical and non-rhythmic auditory stimulation; (3) to the self-selected up-tempo major key song chosen during visit 1 ; and (4) to a metronome beat set at each individual's comfortable tempo. The subject was required to complete one cycle of wrist extension and flexion to each beat. Each condition consisted of 18 15-s trials of wrist flexion and extension, where subjects performed two bimanual trials followed by one unimanual trial with the paretic hand. A
20 -s rest break was provided between each trial to prevent fatigue. The order of the conditions was counterbalanced across subjects, and subjects rated their fatigue levels after the completion of all trials for each condition.

\section{DATA ANALYSIS}

Kinematic data were sampled at $120 \mathrm{~Hz}$ and EMG data were sampled at $1206 \mathrm{~Hz}$. The kinematic data were low pass filtered at $6 \mathrm{~Hz}$ and up-scaled to $1206 \mathrm{~Hz}$ using linear interpolation. The EMG data were filtered using a dual band pass filter (10-52.5 and $67.5-500 \mathrm{~Hz}$ ) and the root mean square (RMS) of the signal was obtained for wrist flexion and extension phases of the movement separately. The EMG signals were normalized to the maximum amplitude recorded for each muscle across all trials and conditions $(46,47)$ to facilitate within- and between-subject comparisons. This method was chosen after extensive reliability testing of different methods of normalization (by Ying Lu). Movement speed, amplitude of wrist extension, wrist extensor activation (RMS of agonist, ECRL), wrist flexor activation (RMS of antagonist, FCU) during extension, and co-activation (defined as RMS of antagonist, FCU/RMS of agonist, ECRL) were the variables used in the analyses. Recognizing that the subjects may present at various stages of recovery at the time of the study, we used hierarchical cluster analysis with the Mahalanobis metric (48) based on baseline wrist kinematics to stratify subjects into groups. The stratification scheme corresponded well with recovery characterized by the Fugl-Meyer Scale as shown in the results below. We then fit linear mixed effect models with group interactions and individual random effects to assess: (1) differences among subject clusters, and (2) learning rates across repeated unimanual trials with the paretic hand after bimanual priming with the four auditory conditions. Learning rate on unimanual trials was defined as the slope of the linear trend fit over the six unimanual trials. All the statistical analyses were conducted using R (v. 2.15.1). The R package "lme 4" was used for the mixed effect model estimation. To control for multiple comparisons but preserve statistical power (due to low sample size in the subgroups), we present all results but choose to interpret results with marginal statistical significance $(0.01<p<0.05)$ with caution.

\section{RESULTS}

Our first objective was to characterize the stages of recovery across a disparate group of patients with post-stroke hemiparesis. Since wrist kinematics provide direct, objective, and reliable measures of movement ability in the paretic hand, we used the movement speed and extent of wrist extension from the first trial with the paretic hand under the baseline condition (no auditory cueing) to perform hierarchical cluster analysis (48), which stratified subjects into three distinct groups (Figure 3).

Clinical metrics (Table 1) showed clear differences across the three groups. The Fugl-Meyer scores were lowest in group 1, followed by group 2 , and then group $3(p=0.047)$. Active wrist motion, measured using goniometry separately from the wrist extension task, showed that both wrist flexion and extension were surprisingly lowest in group 2, intermediate in group 1, and highest in group $3(p<0.001)$. Spasticity at the wrist flexors was, however, 


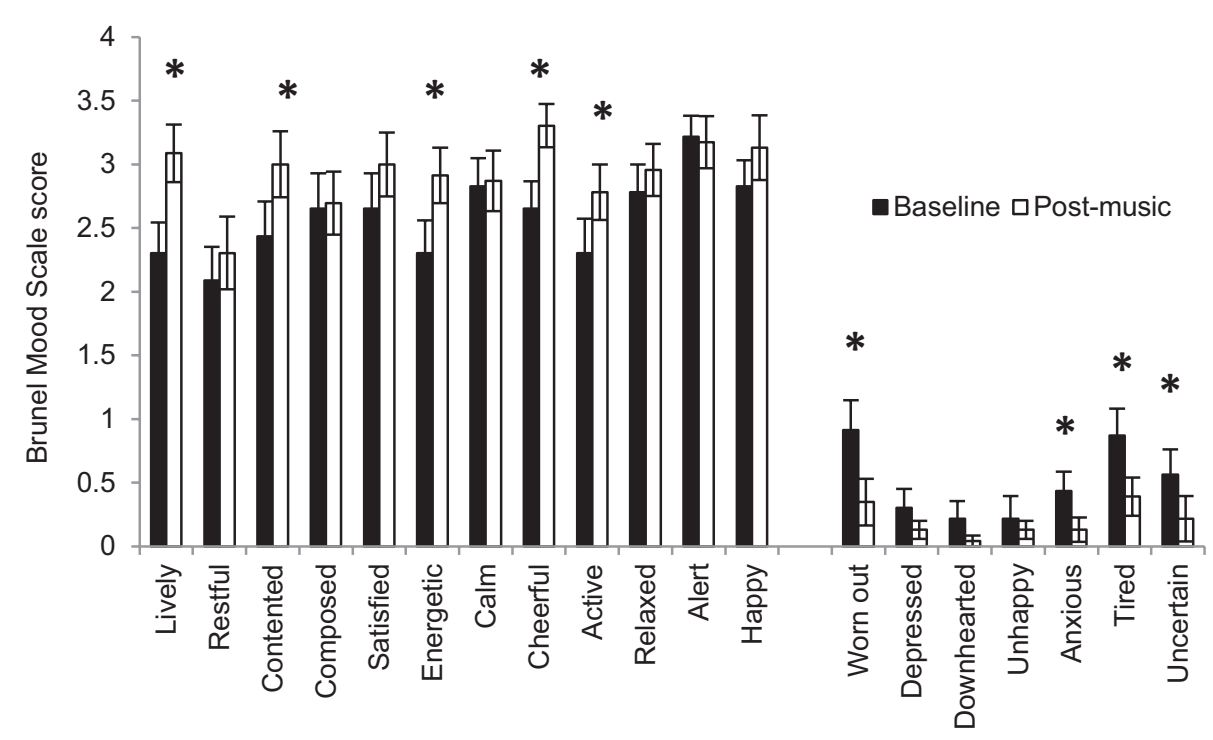

FIGURE 2 | Mean Brunel Mood Scale scores at baseline and after listening to self-selected music. Error bars represent the standard error. ${ }^{*}$ Represents statistically significant differences at $p<0.05$.

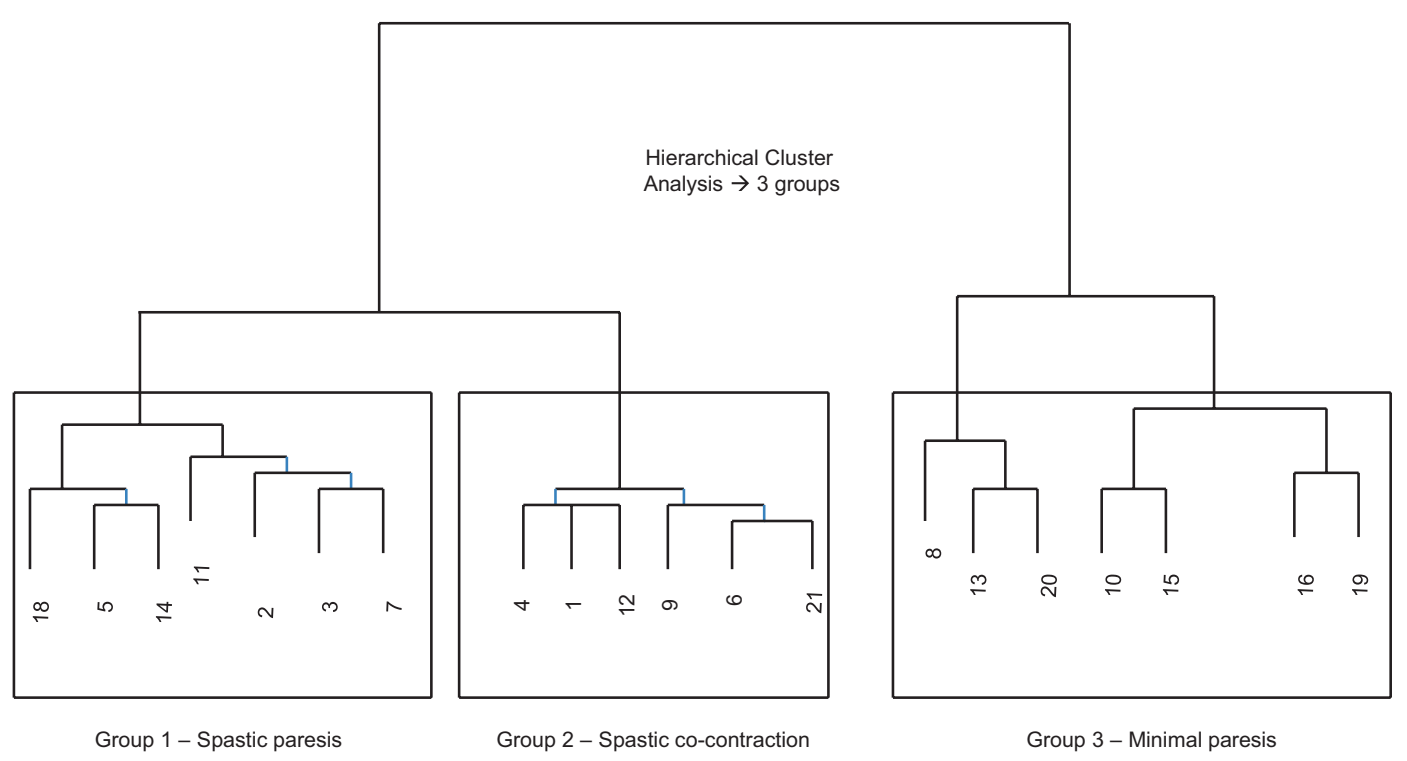

FIGURE 3 | Cluster dendrogram from hierarchical cluster analysis using the Mahalanobis metric based on speed and amplitude of wrist extension on the first trial with the paretic hand at baseline (without auditory stimulation). Three distinct groups emerged.

highest in group 1, and similar in groups 2 and $3(p=0.066)$. There were no significant differences among the three groups in joint proprioception at the wrist, depression scores, mood scores, tempo of the metronome beat or selected song, or fatigue levels. Note that the mean time since stroke was also not different across the three groups $(p=0.89)$.

Baseline performance metrics on the wrist extension task also showed clear differences across the three groups. Movement speed was higher in group 3 compared to groups 1 and $2(p<0.001$, Figure 4A). Extent of wrist extension was lowest in group 2 (where attempted wrist extension produced paradoxical flexion), intermediate in group 1 , and highest in group $3(p<0.001$, Figure 4B). Wrist extensor muscle (ECRL) activation was also lowest in group 2, intermediate in group 1, and highest in group 3 ( $p=0.047$, Figure $4 \mathrm{C})$, whereas wrist flexor muscle (FCU) activation was not differentiated in the three groups $(p=0.877$, Figure 4D). Co-activation between wrist extensor and flexor muscles was highest in group 2, intermediate in group 


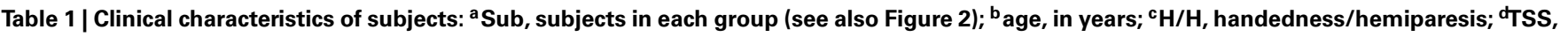

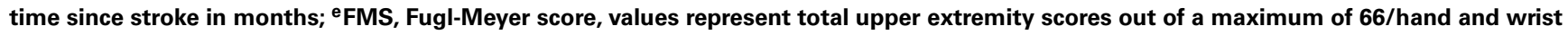
score out of a maximum of 30; ${ }^{\mathrm{f}} \mathrm{AROM}$, active range-of-motion in degrees at the wrist measured with a goniometer; 9 MAS, modified Ashworth Scale measured at the wrist. Lesion location and stroke subtype obtained from: ${ }^{\mathrm{h}}$ radiology reports and ${ }^{\mathrm{i}}$ medical history narrative from subject.

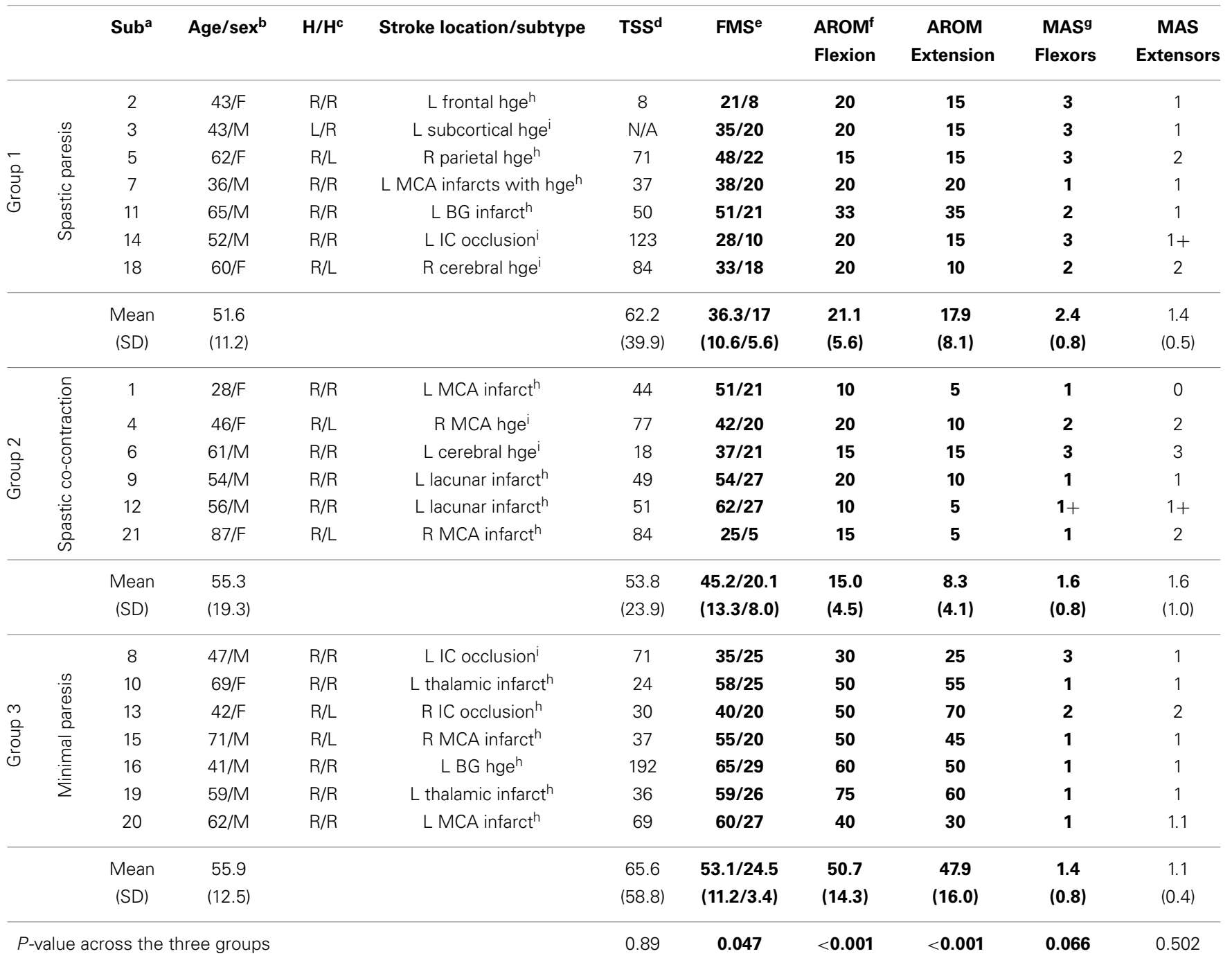

Bolded variables showed statistically and/or clinically important differences across the three groups.

1 , and lowest in group $3(p=0.07$, Figure $4 \mathbf{E})$. Taken together, the baseline performance and clinical metrics enabled characterization of recovery patterns into the three descriptive groups below.

\section{GROUP 1 - SPASTIC PARESIS}

In this group, performance on the paretic side (Figure 4, shown in blue) relative to the non-paretic side showed low movement speed $(\sim 10 \%)$, moderate wrist extension $(\sim 20 \%)$, moderate activation in the wrist extensor $(\sim 30 \%)$, and five times greater coactivation. Clinically, these subjects had the lowest Fugl-Meyer scores (range $21-51$ ), but had $15-33^{\circ}$ of active wrist flexion and $10-35^{\circ}$ of active wrist extension. Spasticity was observed predominantly in the wrist flexors. Lesion location and stroke subtype (Table 1) suggest that these subjects had very severe strokes that were caused predominantly by intracerebral hemorrhage (subject \#s $2,3,5,18$ ) or hemorrhagic transformation of ischemic infarcts (subject \# 7).

\section{GROUP 2 - SPASTIC CO-CONTRACTION}

In this group, performance on the paretic side (Figure 4, shown in red) relative to the non-paretic side showed very slow movement speed $(\sim 7 \%)$, paradoxical wrist flexion on attempted wrist extension $(-27 \%)$, minimal activation of the wrist extensor muscle $(\sim 10 \%)$, and $\sim 10$ times greater co-activation. Clinically, these subjects had higher Fugl-Meyer scores (range 25-62) than those in group 1 . However, they had only $10-20^{\circ}$ of active wrist flexion and 5-15 of active wrist extension. Spasticity was distributed equally in both wrist flexors and extensors for the most part. Lesion location and stroke subtype (Table 1) suggest that these subjects had moderately severe strokes caused predominantly by infarcts in the MCA territory (subject \#s 1, 9, 12, 21). 


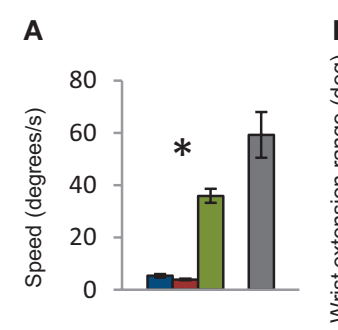

Group 1- Spastic paresis
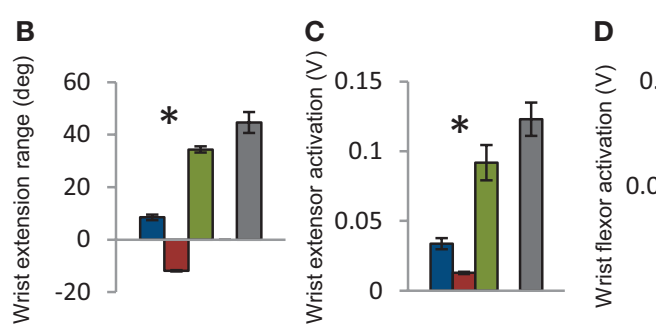

$\mathbf{E}$

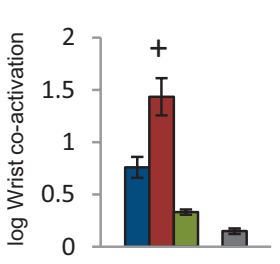

\section{FIGURE 4 | Group means computed for the first trial with the paretic} hand. (A) Speed of wrist extension in degrees per second; (B) extent of wrist extension in degrees; (C) root mean square of wrist extensor muscle activation during wrist extension; (D) root mean square of wrist flexor muscle activation during wrist extension; (E) log of wrist co-activation computed as ratio of wrist flexor to extensor muscle activation. The blue bars represent the group with spastic paresis, which had the lowest

\section{GROUP 3 - MINIMAL PARESIS}

In this group, performance on the paretic side (Figure 4, shown in green) relative to the non-paretic side showed relatively high movement speed $(\sim 60 \%)$, substantial wrist extension $(\sim 77 \%)$, and wrist extensor activation $(\sim 75 \%)$, and twice the co-activation as the non-paretic side. Clinically, these subjects had the highest Fugl-Meyer scores (range 35-65) and the greatest range of active wrist flexion $\left(30-75^{\circ}\right)$ and extension $\left(25-70^{\circ}\right)$ of the three groups. Lesion location and stroke subtype (Table 1) suggest that these subjects had a mixed variety of strokes predominantly in the MCA territory.

Our second objective was to examine how different types of auditory stimuli interact with bimanual training to facilitate subsequent learning with the paretic limb in the three groups. Subjects performed six cycles of two bimanual trials followed by one unimanual trial with the paretic hand, where each trial consisted of multiple repeats of wrist flexion-extension over $15 \mathrm{~s}$. We were interested in the changes in wrist extension, wrist extensor activation, wrist flexor activation, and co-activation over the six unimanual trials for each of the auditory conditions (represented by the different line patterns, see Figure 5). The mean level of the trend lines provides an indication of the amplitude of overall performance, whereas the slope of the trend lines quantifies the rate of learning on the paretic side. A positive slope suggests sustained improvement whereas a negative slope suggests reduced performance under that constraint. Subjects in the spastic paresis (Figure 5A) and spastic co-contraction (Figure 5B) groups started with low or negative wrist extension, but showed sustained improvements under certain auditory constraints. Subjects in the minimal paresis group (Figure 5C), showed good wrist extension at first, but did not improve much over the repeated trials.

The slope (unit change per trial) succinctly summarizes which auditory conditions couple with bimanual training for sustained improvement on the paretic side in the three groups (Figure 6). In the spastic paresis group (Figure 6A), wrist extension improved most with the metronome beat (slope $b=0.86$, $p=0.03)$, even though it also increased wrist flexor activity $(b=0.0021, p<0.0001)$ and co-activation $(b=0.07, p=0.004)$.
Fugl-Meyer scores, the red bars represent the spastic co-contraction group with intermediate Fugl-Meyer scores, and the green bars represent the minimal paresis group, which had the highest Fugl-Meyer scores. Values for the non-paretic hand are shown in gray for reference. Error bars represent the standard error. ${ }^{*}$ Represents differences between the three groups at $p<0.05$, and ${ }^{+}$represents differences between the three groups at $p<0.1$.

Self-selected music did not increase wrist extension, but marginally increased flexor muscle activity $(b=0.0010, p=0.04)$. Thus rhythmic auditory constraints improved motor control in subjects with spastic paresis who were at an earlier stage in motor recovery post-stroke. In the spastic co-contraction group (Figure 6B), wrist extension improved most without any auditory cueing $(b=1.83$, $p<0.0001)$, which increased wrist extensor muscle activation ( $b=0.004, p=0.0002)$ and decreased co-activation across the wrist joint $(b=-0.1, p=0.0006)$. In contrast, self-selected music increased co-activation $(b=0.059, p=0.04)$ in this group. Thus practice without auditory constraints was most beneficial in subjects with spastic co-contraction. In the minimal paresis group (Figure 6C), there was no improvement in wrist extension across the auditory conditions. The slope for wrist extension was most negative with happy sounds $(b=-0.86, p=0.03)$, wrist extensor activation decreased with the metronome beat $(b=-0.0022$, $p=0.02)$, and wrist flexor activation increased without auditory stimulation $(b=0.0012, p=0.015)$.

\section{DISCUSSION}

Neurological and behavioral differences between patients and within each patient over the course of post-stroke recovery can influence how learning occurs during task-specific interactions. Hence, it is necessary to reconcile the vast clinical and movement heterogeneity in the post-stroke population to develop evidencebased rehabilitation protocols directed toward more homogenous groups of patients. Toward this end, the purpose of this study was to: (1) stratify subjects with post-stroke hemiparesis according to their stage of recovery using task-based kinematic measures, and (2) to examine how various types of auditory constraints interact with stage of recovery to facilitate learning of a wrist extension task with the paretic limb. The subjects were stratified into three distinct groups based on their speed and extent of wrist extension. Differences in clinical metrics and task performance led to the characterization of stage of recovery into three groups: (1) the spastic paresis group showed weak extensor drive with flexor spasticity and moderate co-activation of the flexors and extensors, and higher level of motor impairment; (2) the 
A

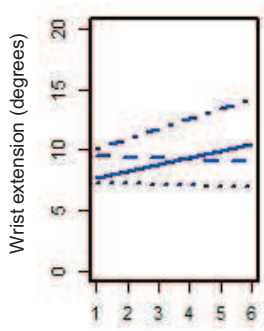

B

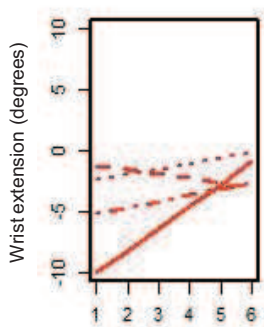

C

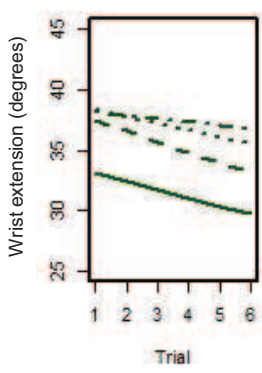

Spastic paresis
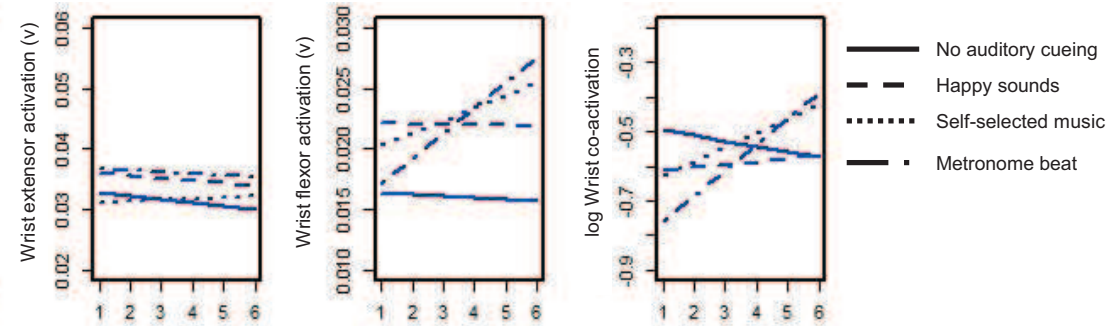

Spastic co-contraction
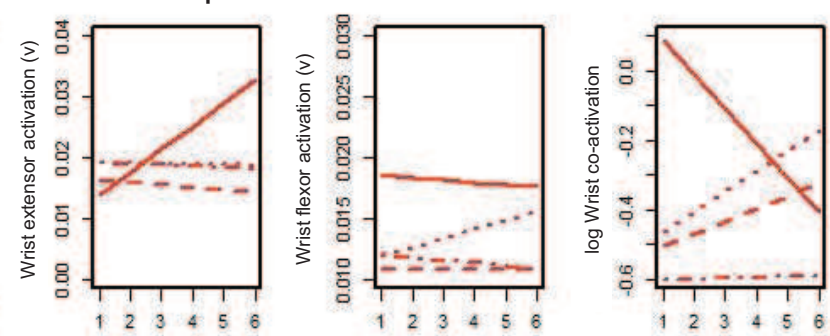

Minimal paresis
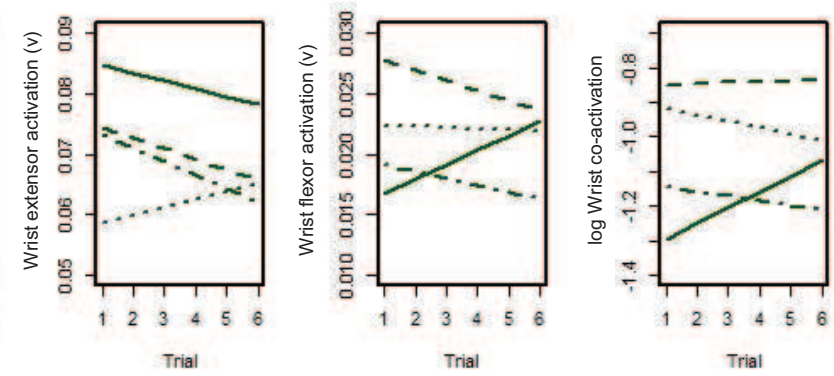

FIGURE 5 | Trendlines of wrist extension performance variables over six repeated trials with the paretic hand under each condition for the three groups: (A) spastic paresis (blue); (B) spastic co-contraction (red); (C) minimal paresis (green). The four conditions are represented by the different patterned lines.

spastic co-contraction group showed higher flexor activation relative to the extensor and excessive co-activation of the flexors and extensors, with moderate level of motor impairment; and (3) the minimal paresis group showed restored extensor drive, low levels of co-activation, and minimal level of motor impairment. The effect of auditory constraints on rate of learning with the paretic hand after bimanual training was measured by the slope of wrist extension, and wrist extensor and flexor muscle activation patterns. Auditory stimulation with a metronome beat increased the rate of learning of wrist extension in subjects with spastic paresis, even though it increased flexor activation and co-activation across the flexor and extensor muscles. In contrast, bimanual training without auditory stimulation produced the greatest improvement in subjects with spastic co-contraction, increased wrist extensor activation, and reduced co-activation. Auditory stimulation in subjects with minimal paresis did not improve wrist extension, but performance was sensitive to the effects of auditory stimulation in this group. These results suggest that altering auditory task constraints during the same task can have different and even opposite effects on motor performance and learning in individuals at different stages of recovery post-stroke. These results cannot be explained by differences in proprioceptive sensation, task difficulty, or fatigue across the groups or conditions. The results further our understanding of possible mechanisms underlying progression of recovery from one stage to the next after stroke.

\section{STRATIFICATION OF SUBJECTS REFLECTS TEMPORAL STAGES IN POST-STROKE RECOVERY}

Subjects with stroke have traditionally been classified based on the time elapsed since their stroke into acute ( $0-3$ months), subacute (3-6 months), and chronic (6 months onward) categories. Recovery has been found to be most rapid in the acute and subacute periods (49), but recently compiled evidence shows that it continues well into the chronic period (50), although the trajectory of recovery may be punctuated by "plateaus" or temporary arrests in recovery. All the subjects in our study were in the chronic phase and may be considered to have plateaued. In longitudinal studies, increases in Fugl-Meyer scores suggest progression toward recovery. The Fugl-Meyer Scale is based on the observation of sequential recovery of motor function by Twitchell and Brunnstrom $(6,9,10)$. It is the most widely used quantitative measure of motor recovery 


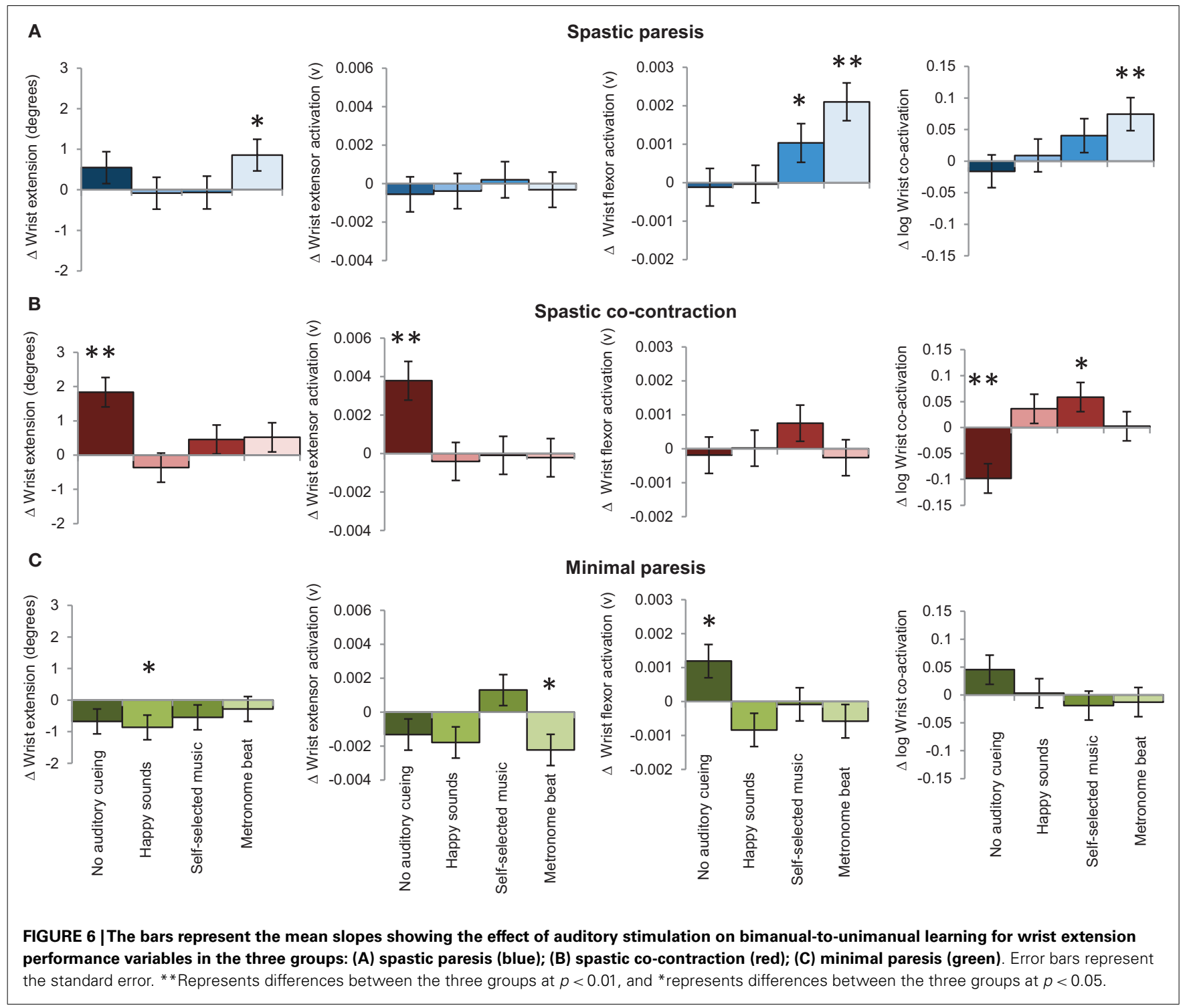

post-stroke $(51,52)$, and the scores have been shown to correlate with the extent of corticospinal tract damage (53). Hence, one can consider subjects with lower Fugl-Meyer scores as being more impaired or at an earlier stage in the recovery process compared to those with higher scores. In this study, subjects in the spastic paresis group had the lowest average Fugl-Meyer scores (both total and for the wrist and hand), which progressively increased in the spastic co-contraction and minimal paresis groups.

Fugl-Meyer scores have also been used to stratify subjects into groups $(54,55)$, but the cut-offs have been variable. Furthermore, the Fugl-Meyer Scale was constructed on the assumptions that recovery proceeds in a proximal-to-distal fashion and from synergistic-to-isolated movements $(8,51)$; however, both these assumptions have been contested recently (56-58). To circumvent the shortcomings of the Fugl-Meyer Scale in stratifying subjects, we used task-based kinematic measures, that is, speed and extent of wrist extension during the task, as direct, objective, and reliable measures of movement ability to stratify subjects into groups. Note that wrist movement amplitudes recorded during the task were lower than those recorded with goniometry prior to the task as would be expected due to the repetitive nature of the task.

We found that the spastic paresis group showed higher speed and amplitude of movement than the spastic co-contraction group, even though the Fugl-Meyer scores were higher in the spastic co-contraction group. This may seem surprising and contradictory to the notion of a linear improvement in movement ability over the course of post-stroke recovery. However, Twitchell observed that spasticity or tone continues to increase and reaches a peak before it starts to decrease (6). In this study, we measured spasticity clinically using the Modified Ashworth Scale, and by the extent of co-activation across the flexors and extensors during the task. We found that the spastic co-contraction group had equally increased tone in both the flexors and extensors, and 10 times greater co-activation on the paretic side than on the non-paretic 
side. While some degree of co-activation between the agonist and antagonist muscles is normal during movement, excessive coactivation leads to reduced movement speed and amplitude (59). Therefore, it follows that a progression of recovery from spastic paresis would lead to a dip in movement ability due to increases in co-activation before it begins to improve again as seen across our three groups. Our results suggest that the processes underlying progression of recovery are non-linear, and predict that movement kinematics and muscle activation patterns may worsen as recovery progresses and then get better. These predictions should be confirmed by future longitudinal studies that measure kinematics and EMG over time.

Furthermore, our results show that auditory constraints increase movement amplitude but also increase muscle coactivation in subjects with spastic paresis, suggesting that individuals at earlier stages of motor recovery benefit from an excitatory drive. In contrast, in subjects with spastic co-contraction, who were at a later stage in recovery and showed excessive co-activation from excitatory overdrive, auditory constraints were not helpful. Instead, bimanual-to-unimanual training without auditory stimulation led to reduced muscle co-activation and increased agonist muscle activity, suggesting that an inhibitory drive may be more beneficial to transition from spastic co-contraction. These findings are discussed further in the sections below. Thus, we propose that stratification of subjects based on relatively simple kinematic parameters of speed and extent of movement into the groups: (1) spastic paresis, (2) spastic co-contraction, and (3) minimal paresis reflects temporal stages in the course of post-stroke recovery, and transition from each of these stages may be triggered by specific constraints imposed during training.

\section{RHYTHMIC AUDITORY STIMULATION IMPROVES PERFORMANCE IN INDIVIDUALS WITH SPASTIC PARESIS}

At baseline, subjects with spastic paresis had both weakness and spasticity, defined as velocity-dependent increase in muscle tone at rest (60), as measured by the Modified Ashworth Scale (61). The emergence of spasticity is thought to reflect re-organization of the descending brainstem pathways leading to diffuse and synergistic patterns of movement. Weakness predominates in the early stages of spasticity (62), hence, while subjects in the spastic paresis group could activate their wrist extensor muscle, their range of wrist extension was limited. Spasticity was greater in the flexor muscles, consistent with the emergence of a flexor synergy pattern (10). Co-activation across the flexors and extensors was increased, but not disabling, as it did not hinder wrist extension $(63,64)$. In this group, auditory stimulation with a metronome beat in conjunction with bimanual training led to increased wrist extension, while that with self-selected music and happy sounds did not. However, both the metronome beat and self-selected music increased wrist flexor activation.

Both the metronome beat and self-selected music have rhythmic components; the rhythm was even and constant with a metronome, but uneven and changing with music. Both even and uneven rhythmic stimulation have been shown to increase muscle co-activation (65). The underlying mechanism is thought to be increased excitability of spinal motor neurons via the reticulospinal pathway, with facilitation of the H-reflex response (66,
67). Using functional MRI and effective connectivity analyzes, it has been shown that listening to music relative to scrambled musical sounds, activates a bilateral network of mesolimbic structures including the nucleus accumbens and the ventral tegmental area (38) leading to dopamine release and arousal. The ventral tegmental area in turn forms part of the midbrain reticular formation where the reticulospinal tracts originate. Excitation of the reticular formation is known to increase spasticity via the reticulospinal projections to the spinal cord (68). Thus, both the metronome beat and stimulating music can increase muscle tone and co-activation that may be helpful in earlier stages of recovery from flaccid paralysis. Non-musical and non-rhythmic auditory stimulation, as in our happy sounds condition, does not produce this effect. Furthermore the type of music, whether stimulating or relaxing, can modulate the extent of arousal and may produce a different effect on muscle tone.

However, only auditory stimulation with a metronome beat in conjunction with bimanual training led to increased unimanual wrist extension, while that with self-selected music and happy sounds did not. Even rhythms have been shown to reduce the variability in EMG responses, whereas uneven rhythms increase the variability in healthy individuals (65). Patients with stroke show disordered motor unit recruitment on EMG (69-72), but training to even metronome beats has been shown to decrease EMG variability (73) and improve motor outcomes post-stroke (23, 25, 27, 73-75). More efficient motor unit recruitment and sensorimotor synchronization (28) to the even metronome beat can explain the increased wrist extension without a notable increase in extensor activation as seen in our subjects with spastic paresis. In contrast, the variable rhythms in music and subtle differences in the type of music chosen, the tempo of the song and its match to the individual's physical abilities may have influenced attention to the rhythmic component of music leading to a reduced peripheral synchronizing effect on wrist extension.

In healthy individuals, sensorimotor coupling to temporally structured auditory input has been shown to recruit a striatothalamo-cortical-system involving basal ganglia, thalamus, premotor cortex (PMC), supplementary motor area (SMA), and dorsolateral prefrontal cortex [see Ref. (76) for review]. Simultaneous bimanual rhythmic movements involve interhemispheric coupling primarily in the PMC, posterior parietal cortex, and cerebellum (77), and switching from simultaneous bimanual synchronized movements to unimanual movements leads to a higher degree of interhemispheric connectivity involving the PMC, SMA, and sensorimotor areas (78). Furthermore, studying acallosal patients has shown that temporal coupling during rhythmic movements arises in large part from interactions between the two hemispheres (79). Taken together with these data, our results suggest that bimanual-to-unimanual movements synchronized to rhythmic auditory stimulation excites a bilateral distributed sensorimotor network, which may facilitate the progression of motor recovery in individuals with spastic paresis.

\section{AUDITORY STIMULATION DOES NOT IMPROVE INHIBITORY CONTROL IN INDIVIDUALS WITH SPASTIC CO-CONTRACTION}

When the threshold for reflex activity continues to reduce due to progressive re-organization of the supraspinal descending drive 
to the spinal cord, peripheral structures of the muscle, muscle spindles, and fascia are further shortened and spasticity evolves into stretch-sensitive forms such as spastic co-contraction (63). Spastic co-contraction refers to inappropriate antagonist recruitment triggered by volitional command (64). Clinically, spastic co-contraction opposes voluntary movement and contributes to impairment in active function, which was seen clearly in our subjects in this group where attempted wrist extension produced paradoxical wrist flexion. While some degree of co-activation between the agonist and antagonist muscles is normal during movement and necessary for joint stability, better movement accuracy and energy efficiency during functional activities, it has been shown to decrease with skill training (80-82). However, its persistence post-stroke signals disrupted reciprocal inhibition of antagonist muscles (83). Sensory feedback from muscle afferents mediates reciprocal inhibition through both spinal and cortical mechanisms $(84,85)$. Cortical suppression of the antagonist muscle is initiated centrally during preparation of agonist muscle contraction $(86,87)$ and the degree of suppression is proportional to the amplitude of stretch of the muscle (88).

Bilateral synchronous mirror symmetric flexion-extension movements have been shown to modulate cortical inhibition in neurologically intact individuals (89) and subjects with stroke (30). Somatosensory and visual information from each side of the body is processed bilaterally (90-92), and interlimb coordination is mediated by motor representations in the parietal and premotor areas shared by both limbs (93). Transcallosal pathways between homotopic regions of the two hemispheres (94-96) may also facilitate transmission of accurate sensory information from the intact hemisphere (33). Passive wrist extension on the affected side (which was facilitated by linked movements with the unaffected hand in this study) in severely impaired patients has been shown to produce fMRI changes in contralesional secondary sensorimotor areas in the ventral premotor and parietal cortices (97), which play a crucial role in re-organization of motor output. Thus, in patients with spastic co-contraction, bimanual training without auditory stimulation may restore sensory feedback, and reinstate reciprocal control in the paretic hand, aiding progression to the next stage of post-stroke recovery. In contrast, self-selected music may have continued to potentiate the stretch reflex through facilitation of descending spinal pathways in this group as discussed above.

\section{INDIVIDUALS WITH MINIMAL PARESIS SHOW VARIED RESPONSES TO AUDITORY STIMULATION}

In subjects with minimal paresis, there was little change in wrist extension across the auditory conditions perhaps due to a ceiling effect. Later stages of recovery have been shown to be mediated by re-organization in the ipsilesional cortex (16-18). Thus, it is not surprising that subjects in this group, who were farther along in their recovery, did not benefit substantially from either bimanual-to-unimanual training or auditory stimulation at the wrist. These strategies would perhaps still be applicable for training of hand and finger control. Subjects with minimal paresis no longer had significant spasticity or co-contraction, but were clearly still impaired compared to the unaffected side. The challenge in these subjects is fine-tuning of muscular control and restoration of dexterity, which may require different types of task constraint.

\section{CONCLUSION}

This was a single-session study where bimanual-to-unimanual training of the paretic side was focused on improvement in performance and learning of a wrist extension task, as restoration of control at the wrist is especially challenging after stroke and necessary for hand function. The main purpose and novelty of this study is to show that auditory stimulation interacts with stage of recovery post-stroke to influence motor learning on a bimanual-to-unimanual wrist extension task. Several important conclusions may be drawn from this study. First, subjects in the chronic post-stroke period can be stratified based on simple movement kinematics to reflect their temporal stage of recovery, which may not be reflected by the time since stroke, and which in turn can inform the selection of strategies to drive subsequent progression of recovery post-stroke. Our data predict that during natural progression of post-stroke recovery, there could be a dip in movement ability due to increased co-contraction and then an increase in movement ability when co-contraction is inhibited. Second, our results show how different auditory constraints influence motor performance at various stages of recovery, perhaps through excitation and inhibition of distinct neural substrates. The effects of auditory constraints on muscle activation patterns provide insight into the mechanisms of transition across impairment levels, contributing to the understanding of how re-organization of CNS pathways may occur. Third, bimanual-to-unimanual learning can be a useful model to probe the rate of learning during singlesession studies, providing an alternative to or a stratification tool prior to lengthy and expensive randomized control trials. We have recently found that long-term training locks-in the transient improvement seen during single-session bimanual-to-unimanual training (Preeti Raghavan, unpublished data). Together, the results lay the foundation for personalized protocols for post-stroke rehabilitation to advance the progression of recovery from one stage to the next, and hold significant implications for further research and clinical practice. Future work may confirm the effect of auditory constraints seen in our study on longitudinal progression of motor recovery in patients at different stages of recovery.

\section{ACKNOWLEDGMENTS}

The authors are grateful to Dr. Concetta Tomaino for her guidance in music selection, and to Aaron Beattie, Barbara Caplan, Puneet Dhaliwal, Isha Doshi, Akash Goyal, Eric Greenwald, Craig Grossman, Benjamin Bonte, and Asad Siddiqi for their assistance with data collection and analysis. Funding: NIH grants T35AT005933 (Alan Leung); K23HD049472 and R01HD071978 (Preeti Raghavan).

\section{REFERENCES}

1. Go AS, Mozaffarian D, Roger VL, Benjamin EJ, Berry JD, Borden WB, et al. Heart disease and stroke statistics - 2013 update: a report from the American Heart Association. Circulation (2013) 127:e6-245. doi:10.1161/CIR. 0b013e318282ab8f

2. Truelsen T, Piechowski-Jozwiak B, Bonita R, Mathers C, Bogousslavsky J, Boysen G. Stroke incidence and prevalence in Europe: a review of available data. Eur J Neurol (2006) 13:581-98. doi:10.1111/j.1468-1331.2006.01138.x 
3. Sousa RM, Ferri CP, Acosta D, Albanese E, Guerra M, Huang Y, et al. Contribution of chronic diseases to disability in elderly people in countries with low and middle incomes: a 10/66 Dementia Research Group population-based survey. Lancet (2009) 374:1821-30. doi:10.1016/S0140-6736(09)61829-8

4. Young J, Forster A. Review of stroke rehabilitation. BMJ (2007) 334:86-90. doi:10.1136/bmj.39059.456794.68

5. Bowden MG, Woodbury ML, Duncan PW. Promoting neuroplasticity and recovery after stroke: future directions for rehabilitation clinical trials. Curr Opin Neurol (2013) 26:37-42. doi:10.1097/WCO.0b013e32835c5ba0

6. Twitchell TE. The restoration of motor function following hemiplegia in man. Brain (1951) 74:443-80. doi:10.1093/brain/74.4.443

7. Brunnstrom S. Associated reactions of the upper extremity in adult patients with hemiplegia: an approach to training. Phys Ther Rev (1956) 36:225-36.

8. Fugl-Meyer AR, Jaasko L, Leyman I, Olsson S, Steglind S. The post-stroke hemiplegic patient. 1. A method for evaluation of physical performance. Scand J Rehabil Med (1975) 7:13-31.

9. Brunnstrom S. Motor testing procedures in hemiplegia: based on sequential recovery stages. Phys Ther (1966) 46:357-75.

10. Brunnstrom S. Movement Therapy in Hemiplegia: A Neurophysiological Approach. New York, NY: Harper \& Row (1970)

11. Grefkes C, Ward NS. Cortical reorganization after stroke: how much and how functional? Neuroscientist (2013) 20:56-70. doi:10.1177/1073858413491147

12. Biernaskie J, Szymanska A, Windle V, Corbett D. Bi-hemispheric contribution to functional motor recovery of the affected forelimb following focal ischemic brain injury in rats. Eur J Neurosci (2005) 21:989-99. doi:10.1111/j.1460-9568. 2005.03899.x

13. Brus-Ramer M, Carmel JB, Martin JH. Motor cortex bilateral motor representation depends on subcortical and interhemispheric interactions. J Neurosci (2009) 29:6196-206. doi:10.1523/JNEUROSCI.5852-08.2009

14. Calautti C, Baron JC. Functional neuroimaging studies of motor recovery after stroke in adults: a review. Stroke (2003) 34:1553-66. doi:10.1161/01.STR. 0000071761.36075.A6

15. Nishimura Y, Onoe H, Morichika Y, Perfiliev S, Tsukada H, Isa T. Timedependent central compensatory mechanisms of finger dexterity after spinal cord injury. Science (2007) 318:1150-5. doi:10.1126/science.1147243

16. Cramer SC. Repairing the human brain after stroke: I. Mechanisms of spontaneous recovery. Ann Neurol (2008) 63:272-87. doi:10.1002/ana.21393

17. Marshall RS, Perera GM, Lazar RM, Krakauer JW, Constantine RC, DeLaPaz RL. Evolution of cortical activation during recovery from corticospinal tract infarction. Stroke (2000) 31:656-61. doi:10.1161/01.STR.31.3.656

18. Ward NS, Brown MM, Thompson AJ, Frackowiak RS. Neural correlates of outcome after stroke: a cross-sectional fMRI study. Brain (2003) 126:1430-48. doi:10.1093/brain/awg245

19. Rehme AK, Fink GR, von Cramon DY, Grefkes C. The role of the contralesional motor cortex for motor recovery in the early days after stroke assessed with longitudinal FMRI. Cereb Cortex (2011) 21:756-68. doi:10.1093/cercor/bhq140

20. Rehme AK, Eickhoff SB, Rottschy C, Fink GR, Grefkes C. Activation likelihood estimation meta-analysis of motor-related neural activity after stroke. Neuroimage (2012) 59:2771-82. doi:10.1016/j.neuroimage.2011.10.023

21. Calautti C, Naccarato M, Jones PS, Sharma N, Day DD, Carpenter AT, et al. The relationship between motor deficit and hemisphere activation balance after stroke: a 3T fMRI study. Neuroimage (2007) 34:322-31. doi:10.1016/j. neuroimage.2006.08.026

22. Chelette KC, Carrico C, Nichols L, Sawaki L. Long-term cortical reorganization following stroke in a single subject with severe motor impairment. Neurorehabilitation (2013) 33(3):385-9. doi:10.3233/NRE- 130968

23. Luft AR, McCombe Waller S, Whitall J, Forrester LW, Macko R, Sorkin JD, et al. Repetitive bilateral arm training and motor cortex activation in chronic stroke: a randomized controlled trial. JAMA (2004) 292:1853-61. doi:10.1001/jama.292.15.1853

24. Whitall J, Waller SM, Sorkin JD, Forrester LW, Macko RF, Hanley DF, et al. Bilateral and unilateral arm training improve motor function through differing neuroplastic mechanisms: a single-blinded randomized controlled trial. Neurorehabil Neural Repair (2011) 25:118-29. doi:10.1177/ 1545968310380685

25. Malcolm MP, Massie C, Thaut M. Rhythmic auditory-motor entrainment improves hemiparetic arm kinematics during reaching movements: a pilot study. Top Stroke Rehabil (2009) 16:69-79. doi:10.1310/tsr1601-69
26. Sarkamo T, Tervaniemi M, Laitinen S, Forsblom A, Soinila S, Mikkonen M, et al. Music listening enhances cognitive recovery and mood after middle cerebral artery stroke. Brain (2008) 131:866-76. doi:10.1093/brain/awn013

27. Thaut MH, Kenyon GP, Hurt CP, McIntosh GC, Hoemberg V. Kinematic optimization of spatiotemporal patterns in paretic arm training with stroke patients. Neuropsychologia (2002) 40:1073-81. doi:10.1016/S0028-3932(01) 00141-5

28. Thaut MH, Kenyon GP, Schauer ML, McInthosh GC. The connection between rhythmicity and brain function. IEEE Eng Med Biol Mag (1999) 18:101-8. doi:10.1109/51.752991

29. Neva JL, Legon W, Staines WR. Primary motor cortex excitability is modulated with bimanual training. Neurosci Lett (2012) 514:147-51. doi:10.1016/j.neulet. 2012.02.075

30. Stinear CM, Barber PA, Coxon JP, Fleming MK, Byblow WD. Priming the motor system enhances the effects of upper limb therapy in chronic stroke. Brain (2008) 131:1381-90. doi:10.1093/brain/awn051

31. Stinear JW, Byblow WD. Rhythmic bilateral movement training modulates corticomotor excitability and enhances upper limb motricity poststroke: a pilot study. J Clin Neurophysiol (2004) 21:124-31. doi:10.1097/00004691200403000-00008

32. Stoykov ME, Stinear JW. Active-passive bilateral therapy as a priming mechanism for individuals in the subacute phase of post-stroke recovery: a feasibility study. Am J Phys Med Rehabil (2010) 89:873-8. doi:10.1097/PHM. 0b013e3181f1c31c

33. Raghavan P, Krakauer JW, Gordon AM. Impaired anticipatory control of fingertip forces in patients with a pure motor or sensorimotor lacunar syndrome. Brain (2006) 129:1415-25. doi:10.1093/brain/awl070

34. Molinari M, Leggio MG, De Martin M, Cerasa A, Thaut M. Neurobiology of rhythmic motor entrainment. Ann N Y Acad Sci (2003) 999:313-21. doi:10.1196/annals.1284.042

35. Thaut MH. Neural basis of rhythmic timing networks in the human brain. Ann N Y Acad Sci (2003) 999:364-73. doi:10.1196/annals.1284.044

36. Lane AM, Crone-Grant D, Lane H. Mood changes following exercise. Percept Mot Skills (2002) 94:732-4. doi:10.2466/pms.2002.94.3.732

37. Marshall RS, Zarahn E, Alon L, Minzer B, Lazar R, Krakauer JW. Early imaging correlates of subsequent motor recovery after stroke. Ann Neurol (2009) 65:596-602. doi:10.1002/ana.21636

38. Menon V, Levitin DJ. The rewards of music listening: response and physiological connectivity of the mesolimbic system. Neuroimage (2005) 28:175-84. doi:10.1016/j.neuroimage.2005.05.053

39. Tschacher W, Schildt M, Sander K. Brain connectivity in listening to affective stimuli: a functional magnetic resonance imaging (fMRI) study and implications for psychotherapy. Psychother Res (2010) 20:576-88. doi:10.1080/ 10503307.2010.493538

40. Parker VM, Wade DT, Langton Hewer R. Loss of arm function after stroke: measurement, frequency, and recovery. Int Rehabil Med (1986) 8: 69-73.

41. Renner CI, Bungert-Kahl P, Hummelsheim H. Change of strength and rate of rise of tension relate to functional arm recovery after stroke. Arch Phys Med Rehabil (2009) 90:1548-56. doi:10.1016/j.apmr.2009.02.024

42. Bohannon RW, Smith MB. Interrater reliability of a modified Ashworth Scale of muscle spasticity. Phys Ther (1987) 67:206-7.

43. Gajdosik RL, Bohannon RW. Clinical measurement of range of motion. Review of goniometry emphasizing reliability and validity. Phys Ther (1987) 67:1867-72.

44. Roger PR, Johnson-Greene D. Comparison of assessment measures for post-stroke depression. Clin Neuropsychol (2009) 23:780-93. doi:10.1080/ 13854040802691135

45. Sheikh JI, Yesavage JA. Geriatric Depression Scale (GDS): recent evidence and development of a shorter version. In: Brink TL, editor. Clinical Gerontology: A Guide to Assessment and Intervention. New York, NY: The Haworth Press (1986). p. 165-73.

46. Burden A. How should we normalize electromyograms obtained from healthy participants? What we have learned from over 25 years of research. J Electromyogr Kinesiol (2010) 20:1023-35. doi:10.1016/j.jelekin.2010.07.004

47. Knutson LM, Soderberg GL, Ballantyne BT, Clarke WR. A study of various normalization procedures for within day electromyographic data. J Electromyogr Kinesiol (1994) 4:47-59. doi:10.1016/1050-6411(94)90026-4 
48. Hines CJ, Selvin S, Samuels SJ, Hammond SK, Woskie SR, Hallock MF, et al. Hierarchical cluster analysis for exposure assessment of workers in the Semiconductor Health Study. Am J Ind Med (1995) 28:713-22. doi:10.1002/ajim. 4700280607

49. Jørgensen HS, Nakayama H, Raaschou HO, Vive-Larsen J, Støier M, Olsen TS. Outcome and time course of recovery in stroke. Part II: time course of recovery. The copenhagen stroke study. Arch Phys Med Rehabil (1995) 76:406-12.

50. Teasell R, Mehta S, Pereira S, McIntyre A, Janzen S, Allen L, et al. Time to rethink long-term rehabilitation management of stroke patients. Top Stroke Rehabil (2012) 19:457-62. doi:10.1310/tsr1906-457

51. Gladstone DJ, Danells CJ, Black SE. The Fugl-Meyer assessment of motor recovery after stroke: a critical review of its measurement properties. Neurorehabil Neural Repair (2002) 16:232-40. doi:10.1177/154596802401105171

52. van Wijck FM, Pandyan AD, Johnson GR, Barnes MP. Assessing motor deficits in neurological rehabilitation: patterns of instrument usage. Neurorehabil Neural Repair (2001) 15:23-30. doi:10.1177/154596830101500104

53. Zhu LL, Lindenberg R, Alexander MP, Schlaug G. Lesion load of the corticospinal tract predicts motor impairment in chronic stroke. Stroke (2010) 41:910-5. doi:10.1161/STROKEAHA.109.577023

54. Michaelsen SM, Luta A, Roby-Brami A, Levin MF. Effect of trunk restraint on the recovery of reaching movements in hemiparetic patients. Stroke (2001) 32:1875-83. doi:10.1161/01.STR.32.8.1875

55. Pang MY, Harris JE, Eng JJ. A community-based upper-extremity group exercise program improves motor function and performance of functional activities in chronic stroke: a randomized controlled trial. Arch Phys Med Rehabil (2006) 87:1-9. doi:10.1016/j.apmr.2005.08.113

56. Beebe JA, Lang CE. Absence of a proximal to distal gradient of motor deficits in the upper extremity early after stroke. Clin Neurophysiol (2008) 119:2074-85. doi:10.1016/j.clinph.2008.04.293

57. Crow JL, Harmeling-van der Wel BC. Hierarchical properties of the motor function sections of the Fugl-Meyer Assessment Scale for people after stroke: a retrospective study. Phys Ther (2008) 88:1554-67. doi:10.2522/ptj. 20070186

58. Woodbury ML, Velozo CA, Richards LG, Duncan PW, Studenski S, Lai SM. Dimensionality and construct validity of the Fugl-Meyer assessment of the upper extremity. Arch Phys Med Rehabil (2007) 88:715-23. doi:10.1016/j.apmr. 2007.02.036

59. Rosa MC, Marques A, Demain S, Metcalf CD. Lower limb co-contraction during walking in subjects with stroke: a systematic review. J Electromyogr Kinesiol (2013) 24(1):1-10. doi:10.1016/j.jelekin.2013.10.016

60. Lance JW, editor. Symposium Synopsis. Chicago, IL: Year Book Medical Publishers (1980).

61. Katz RT, Rymer WZ. Spastic hypertonia: mechanisms and measurement. Arch Phys Med Rehabil (1989) 70:144-55.

62. Burke D. Spasticity as an adaptation to pyramidal tract injury. Adv Neurol (1988) 47:401-23.

63. Gracies J-M. Pathophysiology of spastic paresis. I: paresis and soft tissue changes. Muscle Nerve (2005) 31:535-51. doi:10.1002/mus.20284

64. Gracies JM. Pathophysiology of spastic paresis. II: emergence of muscle overactivity. Muscle Nerve (2005) 31:552-71. doi:10.1002/mus.20285

65. Safranek MG, Koshland GF, Raymond G. Effect of auditory rhythm on muscle activity. Phys Ther (1982) 62:161-8.

66. Paltsev YI, Elner AM. Change in the functional state of the segmental apparatus of the spinal cord under the influence of sound stimuli and its role in voluntary movement. Biophysics (1967) 12:1219-26.

67. Rossignol S, Melvill Jones G. Audio-spinal influences in man studied by the H-reflex and its possible role on rhythmic movements synchronized to sound. Electroencephalogr Clin Neurophysiol (1976) 41(1):83-92. doi:10.1016/00134694(76)90217-0

68. Brown P. Pathophysiology of spasticity. J Neurol Neurosurg Psychiatry (1994) 57:773-7. doi:10.1136/jnnp.57.7.773

69. Gowland C, deBruin H, Basmajian JV, Plews N, Burcea I. Agonist and antagonist activity during voluntary upper-limb movement in patients with stroke. Phys Ther (1992) 72:624-33.

70. Hu X, Suresh AK, Li X, Rymer WZ, Suresh NL. Impaired motor unit control in paretic muscle post stroke assessed using surface electromyography: a preliminary report. Conf Proc IEEE Eng Med Biol Soc (2012) 2012:4116-9. doi:10.1109/EMBC.2012.6346872
71. Li X, Suresh A, Zhou P, Rymer WZ. Alterations in the peak amplitude distribution of the surface electromyogram poststroke. IEEE Trans Biomed Eng (2013) 60:845-52. doi:10.1109/TBME.2012.2205249

72. Suresh N, Li X, Zhou P, Rymer WZ. Examination of motor unit control properties in stroke survivors using surface EMG decomposition: a preliminary report. Conf Proc IEEE Eng Med Biol Soc (2011) 2011:8243-6. doi:10.1109/IEMBS.2011.6092032

73. Thaut MH, Leins AK, Rice RR, Argstatter H, Kenyon GP, McIntosh GC, et al. Rhythmic auditory stimulation improves gait more than NDT/Bobath training in near-ambulatory patients early poststroke: a single-blind, randomized trial. Neurorehabil Neural Repair (2007) 21:455-9. doi:10.1177/1545968307300523

74. McCombe Waller S, Liu W, Whitall J. Temporal and spatial control following bilateral versus unilateral training. Hum Mov Sci (2008) 27:749-58. doi:10.1016/j.humov.2008.03.006

75. Whitall J, McCombe Waller S, Silver KH, Macko RF. Repetitive bilateral arm training with rhythmic auditory cueing improves motor function in chronic hemiparetic stroke. Stroke (2000) 31:2390-5. doi:10.1161/01.STR.31.10.2390

76. Repp BH, Su YH. Sensorimotor synchronization: a review of recent research (2006-2012). Psychon Bull Rev (2013) 20:403-52. doi:10.3758/s13423-0120371-2

77. Pollok B, Sudmeyer M, Gross J, Schnitzler A. The oscillatory network of simple repetitive bimanual movements. Brain Res Cogn Brain Res (2005) 25:300-11. doi:10.1016/j.cogbrainres.2005.06.004

78. Serrien DJ. The neural dynamics of timed motor tasks: evidence from a synchronization-continuation paradigm. Eur J Neurosci (2008) 27:1553-60. doi:10.1111/j.1460-9568.2008.06110.x

79. Sternad D, Wei K, Diedrichsen J, Ivry RB. Intermanual interactions during initiation and production of rhythmic and discrete movements in individuals lacking a corpus callosum. Exp Brain Res (2007) 176:559-74. doi:10.1007/ s00221-006-0640-2

80. Arias P, Espinosa N, Robles-Garcia V, Cao R, Cudeiro J. Antagonist muscle co-activation during straight walking and its relation to kinematics: insight from young, elderly and Parkinson's disease. Brain Res (2012) 1455:124-31. doi:10.1016/j.brainres.2012.03.033

81. Floeter MK, Danielian LE, Kim YK. Effects of motor skill learning on reciprocal inhibition. Restor Neurol Neurosci (2013) 31:53-62. doi:10.3233/RNN-120247

82. Milner TE. Adaptation to destabilizing dynamics by means of muscle cocontraction. Exp Brain Res (2002) 143:406-16. doi:10.1007/s00221-0021001-4

83. Gerachshenko T, Rymer WZ, Stinear JW. Abnormal corticomotor excitability assessed in biceps brachii preceding pronator contraction post-stroke. Clin Neurophysiol (2008) 119:683-92. doi:10.1016/j.clinph.2007.11.004

84. Bertolasi L, Priori A, Tinazzi M, Bertasi V, Rothwell JC. Inhibitory action of forearm flexor muscle afferents on corticospinal outputs to antagonist muscles in humans. J Physiol (1998) 511(Pt 3):947-56. doi:10.1111/j.1469-7793.1998. 947bg.x

85. Bertolasi L, Romito S, Tinazzi M, Rizzuto N, Priori A. Impaired heteronymous somatosensory motor cortical inhibition in dystonia. Mov Disord (2003) 18:1367-73. doi:10.1002/mds.10514

86. Gerachshenko T, Stinear JW. Suppression of motor evoked potentials in biceps brachii preceding pronator contraction. Exp Brain Res (2007) 183:531-9. doi:10.1007/s00221-007-1071-4

87. Hoshiyama M, Kitamura Y, Koyama S, Watanabe S, Shimojo M, Kakigi R. Reciprocal change of motor evoked potentials preceding voluntary movement in humans. Muscle Nerve (1996) 19:125-31. doi:10.1002/(SICI)10974598(199602)19:2<125::AID-MUS1>3.0.CO;2-G

88. Coxon JP, Stinear JW, Byblow WD. Amplitude of muscle stretch modulates corticomotor gain during passive movement. Brain Res (2005) 1031:109-17. doi:10.1016/j.brainres.2004.10.062

89. Stinear JW, Byblow WD. Disinhibition in the human motor cortex is enhanced by synchronous upper limb movements. J Physiol (2002) 543:307-16. doi:10. 1113/jphysiol.2002.023986

90. Hinkley LB, Krubitzer LA, Nagarajan SS, Disbrow EA. Sensorimotor integration in S2, PV, and parietal rostroventral areas of the human sylvian fissure. J Neurophysiol (2007) 97:1288-97. doi:10.1152/jn.00733.2006

91. Liu Z, Zhang N, Chen W, He B. Mapping the bilateral visual integration by EEG and fMRI. Neuroimage (2009) 46:989-97. doi:10.1016/j.neuroimage.2009.03. 028 
92. Zhu Z, Disbrow EA, Zumer JM, McGonigle DJ, Nagarajan SS. Spatiotemporal integration of tactile information in human somatosensory cortex. BMC Neurosci (2007) 8:21. doi:10.1186/1471-2202-8-21

93. Ehrsson HH, Naito E, Geyer S, Amunts K, Zilles K, Forssberg H, et al. Simultaneous movements of upper and lower limbs are coordinated by motor representations that are shared by both limbs: a PET study. Eur J Neurosci (2000) 12:3385-98. doi:10.1046/j.1460-9568.2000.00209.x

94. Boussaoud D, Tanne-Gariepy J, Wannier T, Rouiller EM. Callosal connections of dorsal versus ventral premotor areas in the macaque monkey: a multiple retrograde tracing study. BMC Neurosci (2005) 6:67. doi:10.1186/1471-2202-6-67

95. Liu J, Morel A, Wannier T, Rouiller EM. Origins of callosal projections to the supplementary motor area (SMA): a direct comparison between pre-SMA and SMA-proper in macaque monkeys. J Comp Neurol (2002) 443:71-85. doi:10.1002/cne.10087

96. Rouiller EM, Babalian A, Kazennikov O, Moret V, Yu XH, Wiesendanger M. Transcallosal connections of the distal forelimb representations of the primary and supplementary motor cortical areas in macaque monkeys. Exp Brain Res (1994) 102:227-43. doi:10.1007/BF00227511

97. Dechaumont-Palacin S, Marque P, De Boissezon X, Castel-Lacanal E, Carel C, Berry I, et al. Neural correlates of proprioceptive integration in the contralesional hemisphere of very impaired patients shortly after a subcortical stroke: an FMRI study. Neurorehabil Neural Repair (2008) 22:154-65. doi: $10.1177 / 1545968307307118$

Conflict of Interest Statement: The authors declare that the research was conducted in the absence of any commercial or financial relationships that could be construed as a potential conflict of interest.

Received: 01 February 2014; accepted: 10 June 2014; published online: 23 June 2014. Citation: Aluru V, Lu Y, Leung A, Verghese J and Raghavan P (2014) Effect of auditory constraints on motor performance depends on stage of recovery post-stroke. Front. Neurol. 5:106. doi: 10.3389/fneur.2014.00106

This article was submitted to Movement Disorders, a section of the journal Frontiers in Neurology.

Copyright (C) 2014 Aluru, Lu, Leung, Verghese and Raghavan. This is an open-access article distributed under the terms of the Creative Commons Attribution License (CC $B Y)$. The use, distribution or reproduction in other forums is permitted, provided the original author(s) or licensor are credited and that the original publication in this journal is cited, in accordance with accepted academic practice. No use, distribution or reproduction is permitted which does not comply with these terms. 